Methodology article

\title{
Multigene expression of protein complexes by iterative modification of genomic Bacmid DNA Rob J Noad ${ }^{1,3}$, Meredith Stewart ${ }^{1}$, Mark Boyce ${ }^{1}$, Cristina C Celma ${ }^{1}$, Keith R Willison ${ }^{2}$ and Polly Roy*1
}

Address: ${ }^{1}$ Department of Infectious and Tropical Diseases, London School of Hygiene and Tropical Medicine, Keppel Street, London, WC1E 7HT, UK, ${ }^{2}$ Section of Cell and Molecular Biology, Institute of Cancer Research, 237 Fulham Road, London SW3 6JB, UK and ${ }^{3}$ Current address: Department of Pathology and Infectious Diseases, Royal Veterinary College, Hawkshead Lane, Hatfield AL9 7TA, UK

Email: Rob J Noad - rnoad@rvc.ac.uk; Meredith Stewart - meredith.stewart@lshtm.ac.uk; Mark Boyce - mark.boyce@lshtm.ac.uk; Cristina C Celma - cristina.celma@lshtm.ac.uk; Keith R Willison - Keith.Willison@icr.ac.uk; Polly Roy* - Polly.Roy@lshtm.ac.uk

* Corresponding author

Published: 2 September 2009

BMC Molecular Biology 2009, 10:87 doi:10.1 186/147|-2199-10-87
Received: 27 April 2009

Accepted: 2 September 2009

This article is available from: http://www.biomedcentral.com//47/-2/99//0/87

(c) 2009 Noad et al; licensee BioMed Central Ltd.

This is an Open Access article distributed under the terms of the Creative Commons Attribution License (http://creativecommons.org/licenses/by/2.0), which permits unrestricted use, distribution, and reproduction in any medium, provided the original work is properly cited.

\begin{abstract}
Background: Many cellular multi-protein complexes are naturally present in cells at low abundance. Baculovirus expression offers one approach to produce milligram quantities of correctly folded and processed eukaryotic protein complexes. However, current strategies suffer from the need to produce large transfer vectors, and the use of repeated promoter sequences in baculovirus, which itself produces proteins that promote homologous recombination. One possible solution to these problems is to construct baculovirus genomes that express each protein in a complex from a separate locus within the viral DNA. However current methods for selecting such recombinant genomes are too inefficient to routinely modify the virus in this way.

Results: This paper reports a method which combines the lambda red and bacteriophage PI Crerecombinase systems to efficiently generate baculoviruses in which protein complexes are expressed from multiple, single-locus insertions of foreign genes. This method is based on an 88 fold improvement in the selection of recombinant viruses generated by red recombination techniques through use of a bipartite selection cassette. Using this system, seven new genetic loci were identified in the AcMNPV genome suitable for the high level expression of recombinant proteins. These loci were used to allow the recovery two recombinant virus-like particles with potential biotechnological applications (influenza $\mathrm{A}$ virus $\mathrm{HA} / \mathrm{MI}$ particles and bluetongue virus VP2/VP3/VP5/VP7 particles) and the mammalian chaperone and cancer drug target CCT (16 subunits formed from 8 proteins).

Conclusion: I. Use of bipartite selections can significantly improve selection of modified bacterial artificial chromosomes carrying baculovirus DNA. Furthermore this approach is sufficiently robust to allow routine modification of the virus genome. 2 . In addition to the commonly used $p / 0$ and polyhedrin loci, the ctx, egt, 39k, orf5 I, gp37, iap2 and odv-e56 loci in AcMNPV are all suitable for the high level expression of heterologous genes. 3. Two protein, four protein and eight protein complexes including virus-like particles and cellular chaperone complexes can be produced using the new approach.
\end{abstract}




\section{Background}

The baculovirus Autographa califonica multiple nucleopolyhedrosis virus (AcMNPV) is routinely used to express proteins in eukaryotic cells for structural, biochemical and vaccine studies [1]. The AcMNPV genome is circular dsDNA ( 134 $\mathrm{kb})$ and contains regions of highly repetitive DNA elements and genes in both strands [2]. This genomic DNA can be propagated in Escherichia coli as a bacmid, and genes can be inserted by $T n 7$ transposase based transposition into the virus DNA [3]. Transfection of the modified bacmid DNA into virus susceptible insect cells results in recovery infectious virus expressing the recombinant protein corresponding to the inserted gene [3]. Alternate approaches in which homologous recombination is carried out in insect cells can also be used to generate recombinant baculoviruses $[4,5]$.

One of the advantages of the baculovirus system is its utility for the co-expression of multiple genes that encode protein complexes [6-12]. This is important, as many critical functions of living cells are carried out by multi-subunit protein complexes which are naturally present at low abundance. One approach for the baculovirus mediated co-expression of multiple genes is the insertion of expression cassettes as tandem or inverted repeats at the polyhedrin locus in the viral genomic DNA $[6-9,12,13]$. This approach ensures that every cell in the culture expresses the genes encoding recombinant proteins in the same ratio and results in consistent yields of recombinant protein complex [14]. However, this approach has two major limitations. Firstly, there is a practical limit to the number of genes that can be inserted into one transfer vector in terms of vector size. In practice, this means that it is rarely possible to express more than four proteins from a single locus. In addition, baculovirus expresses proteins that promote homologous recombination [15-17], therefore viruses that contain large amounts of repeated sequences, such as common promoters and terminators, are prone to rearrangement and recombination $[11,18,19]$. A potential solution to these problems would be the production of viral genomes in which foreign genes are each expressed from a different genetic locus, removing the need for large transfer vectors with highly repetitive sequences. However, to date, most baculovirus expression experiments have been carried out with one of two loci (polyhedrin and $\mathrm{p} 10)$. There have also been reports of $v$-cath $[10,11]$ being used to express heterologous proteins, but the expression of foreign genes from other loci in the genome is largely uncharacterised. Therefore, before any multilocus approach to express genes for larger complexes could be pursued it would be necessary to characterise foreign gene expression at alternative loci within the AcMNPV genome.

Recently baculovirus research has benefited from use of the lambda red recombination [20] approach for selective knockout of viral genes [21-28]. However, the potential for this technique to engineer high level expression of foreign genes from different genetic loci within the baculovirus genome has not been examined. In this report, we describe high level expression of foreign genes from seven loci within the AcMNPV genome not previously characterised for this purpose. Furthermore, we demonstrate a functional multi-locus system for the expression of multiple foreign genes from a single baculovirus genome. In this system single protein expression cassettes are inserted efficiently at different loci within the viral genome using lambda red recombination. As single-gene insertions are thus distributed throughout the AcMNPV genome, the problems of transfer vector size and highly repetitive inserts are overcome. We demonstrate the utility of this system for the expression of complexes with up to eight subunits using influenza A VLPs (2 proteins), bluetongue VLPs (4 proteins) and the essential mammalian chaperonin complex CCT (8 proteins) as examples.

\section{Results \\ $A$ bipartite selection system allows significant increase in the efficiency of recombinant bacmid recovery}

Lambda red recombination [20] has been widely used to introduce knockout mutations in AcMNPV [21-28]. In the majority of these experiments a chloramphenicol resistance gene has been used as a selectable marker. In our hands, although this system worked, the speed with which experiments could be completed was hampered by the number of false positive colonies arising due to the low levels of antibiotic used in selection of the low copy bacmid. Also, it was not possible to introduce multiple modifications into the virus genome without either accumulating antibiotic resistance genes or performing another round of lambda red recombination and selecting for virus which had lost the selectable marker. To overcome these problems we designed a new bipartite selection cassette based on LacZ $\alpha$ fragment and Zeocin resistance gene flanked by modified loxP recombination sites (Fig. 1A). Using this system, sufficient Zeocin was used to reduce, but not eliminate, background colony growth and recombinants were selected based on blue colony phenotype in the presence of IPTG and X-gal.

To assess the relative efficiency of recovering recombinants using the chloramphenicol and bipartite selections, recombination competent $E$. coli containing unmodified AcMNPV bacmid were electroporated with $30 \mathrm{ng}(\sim 12.5 \mathrm{fmol})$ linear DNA containing each selection cassette (Fig. 1B). The efficiency of recombination using PCR derived and plasmid derived, restriction enzyme released, linear DNA was compared. PCR primers were designed such that the ends of the PCR products corresponded to the ends of the restriction enzyme released fragments. The bipartite selection resulted in a 21 fold increase in the number of positive colonies for PCR 

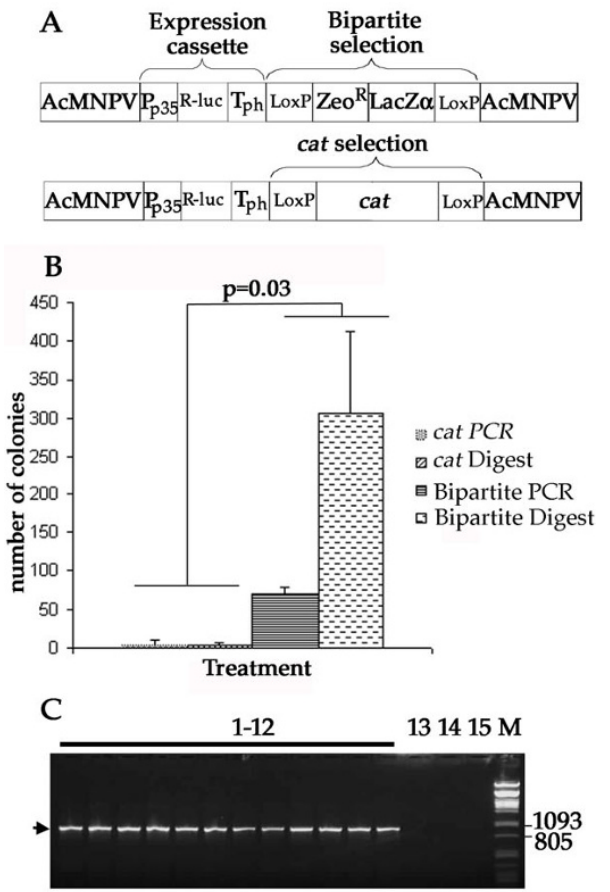

D

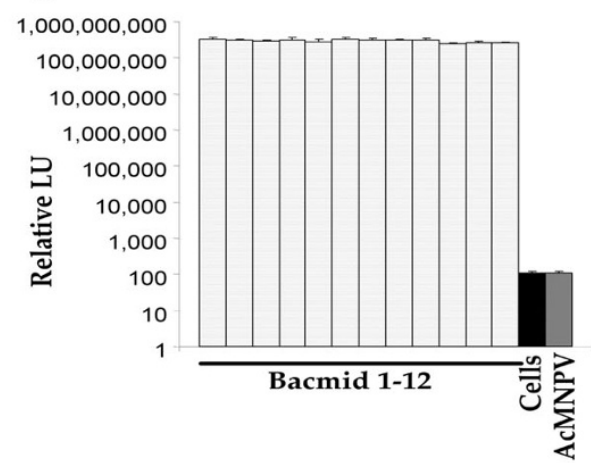

Figure I

Bipartite selection provides significant increase in selection efficiency for lambda red Bacmid recombination. A) Two recombination cassettes were designed, both with baculovirus flanking sequences (AcMNPV), p35 promoter $\left(P_{p 35}\right)$, Renilla Luciferase (R-luc) and polyhedrin polyadenylation sequences $\left(T_{p h}\right)$ and LoxP sites flanking a bacterial selectable marker. For bipartite selection (top) a Zeocin resistance gene (ZeoR) and LacZ $\alpha$ marker were incorporated. For single marker selection (bottom) the cat gene, conferring chloramphenicol resistance, was incorporated. B) The number of positive colonies following red recombination using $30 \mathrm{ng}$ of each recombination cassette $(\sim 12.58 \mathrm{fmol})$, generated either by PCR amplification or restriction enzyme digestion, and BACmid DNA. Each transformation was carried out in triplicate, error bars indicate the standard deviation of the results. The data for the two different selections were clustered and a t-test used to confirm that the results were statistically significant $(p=0.03)$. C) PCR confirming recombination and correct target site 12 independent bacmid recombinants (lanes I-I2). One primer annealed to genomic DNA flanking the target insertion site and the other to sequence inside the recombination cassette. Only recombination at the correct genetic locus would produce the PCR product (arrowed). Lane 13, no template PCR reaction; Lane I4, bMONI4272 Bacmid template DNA; Lane I5 plasmid containing the entire DNA used for recombination as template. D) Renilla luciferase activity at 48 hours post infection in cell lysates from cells infected with passage 2 of recombinant bacmids I-I 2 from B. Cells and AcMNPV indicate background activity in lysates from uninfected and unmodified bacmid infected cells, respectively. 
derived DNA and an 88 fold increase for restriction enzyme released plasmid DNA compared to the single antibiotic selection. These differences were significant ( $t-$ test, $\mathrm{p}=0.03$ ). To avoid repeated PCR of coding sequences, and to take advantage of the increased efficiency of recombination, linear DNA derived by restriction digestion of plasmid DNA was used for subsequent experiments.

PCR was carried out on bacmid DNA purified from positive colonies from the bipartite selection to confirm correct insertion of the expression construct. Primers complementary to the zeocin resistance gene and the AcMNPV DNA flanking the correct insertion site were used. PCR product was only produced where correctly targeted recombination had occurred. DNA for twelve separate bacmids were tested using this method (Fig. 1C, lanes 1-12), all were positive for the PCR product, indicating correctly targeted recombination. In contrast, neither bacmid DNA alone, nor plasmid DNA containing the DNA fragment used for recombination was able to act as template to produce the PCR product (Fig. 1C, lanes 14 and 15 respectively). To test the viability of recombinant baculovirus, the same twelve PCR positive bacmid clones were transfected into $S f 9$ cells and passaged twice, then Renilla luciferase activity in cells infected with each of the recombinants was assayed at 48 hours post infection. Cells infected with each of the twelve recombinant viruses had Renilla luciferase activities that were $10^{6}$ fold above background (Fig. 1D). Based on these data the bipartite selection was used for further studies. The genetic locus used for these initial studies was the $p 10$ locus, which was modified to remove native $p 10$ promoter sequences.

\section{Cre mediated removal of the selectable marker allows multiple rounds of recombination with the same bipartite selection}

In order to engineer multiple insertions in the AcMNPV genome, the bipartite selection cassette was flanked by modified loxP sites. These sites incorporated both the lox66 and lox71 mutations that limit Cre mediated recombination to a single round [29], and a mutation in the spacer reducing homology to the wildtype loxP site. Cre mediated recombination would thus result in the removal of the bipartite selectable marker and inactivation of the lox recombination sites but leave behind the baculovirus expression cassette (Fig. 2A). To confirm this strategy could be used successfully to engineer multiple insertions in the bacmid DNA, Cre recombination was used to remove the bipartite marker from bacmid in which the renilla luciferase reporter had been inserted. Bacmid DNA was purified from four putative recombinants and recombination confirmed by PCR using primers flanking the selectable marker (Fig. 2B). All four PCR products were consistent with the size expected for successful Cre recombination. This was further confirmed by sequencing across the modified loxP site of the recombinants (Fig. 2C). To confirm that Cre mediated bacmid recombinants remained viable in insect cells, bacmid DNA was transfected into insect cells and luciferase activity assayed after two passages as before. All recombinants had renilla luciferase activity that was equivalent to the parental baculovirus before Cre recombination (Fig. 2D).

\section{Identification of genetic loci in the baculovirus genome suitable for high level expression of heterologous genes} To test whether the new selection could be used efficiently at different baculovirus genetic loci a second round of recombination was carried out using one of the bacmids already containing the renilla luciferase gene. A second reporter, firefly luciferase, under control of the polyhedrin promoter was inserted at a total of thirteen different genetic loci (ctx, orf11, egt, orf $23, v-f g f, 39 k$, orf51, gp 37 , iap2, chiA, pe, odv-e18 and odv-e56), in each case generating a dual expression baculovirus for Renilla and firefly luciferase (Fig. 3A). Additional changes were made at certain loci to specifically inactivate the expression of the endogenous viral gene (Fig. 3B). Recombinant viruses were passaged twice in $S f 9$ insect cells and on the third passage cells were harvested at 48 hours post infection, lysed and assayed for both firefly and Renilla luciferase activities. Renilla luciferase activity was used as a reference for virus replication and protein expression as all the recombinants had the same P35 promoter driven Renilla luciferase cassette in the $p 10$ locus. Expression of firefly luciferase at each locus was compared to a virus carrying firefly luciferase gene at the polyhedrin locus. Of the thirteen loci tested, nine had renilla luciferase activities which were at least $10^{5}$ fold above background, and of these eight (ctx, egt, orf51, gp37, iap2, chiA, odv-e18 and odv-e56) had renilla luciferase activity which was equivalent to that of the parental renilla luciferase only control (Fig. 3C). Thus there was no evidence of a general impairment in virus replication or gene expression for these viruses. In contrast, four insertions (orf 11, $v$-fgf, pe and orf23) resulted in virus infections where renilla luciferase activity was within 10 fold of the background and were exluded from further analysis.

Renilla luciferase activity was used as a reference to normalise firefly luciferase activity and obtain a measure of relative expression of the firefly luciferase from each locus (Fig. 3D). Seven loci (ctx, egt, 39k, orf51, gp37, iap2 and $o d v$-e56) had firefly luciferase activity which was at least $10^{6}$ fold higher than background and similar to polyhedrin locus expression of the gene (Fig. 3D). Two viruses (chiA and odv-e18) had high levels of renilla luciferase but relatively poor firefly luciferase activity suggesting that although these viruses were capable of replication, expression of the polyhedrin promoter driven reporter at this locus was impaired. 


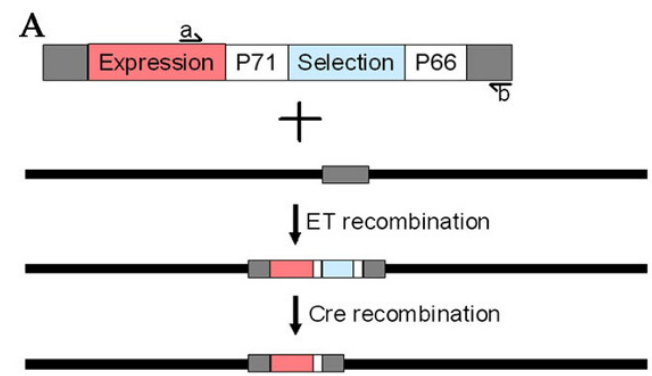

B

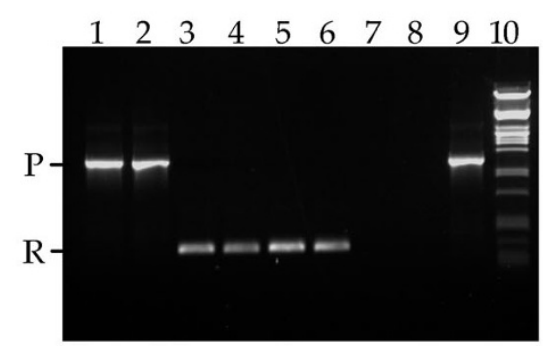

C
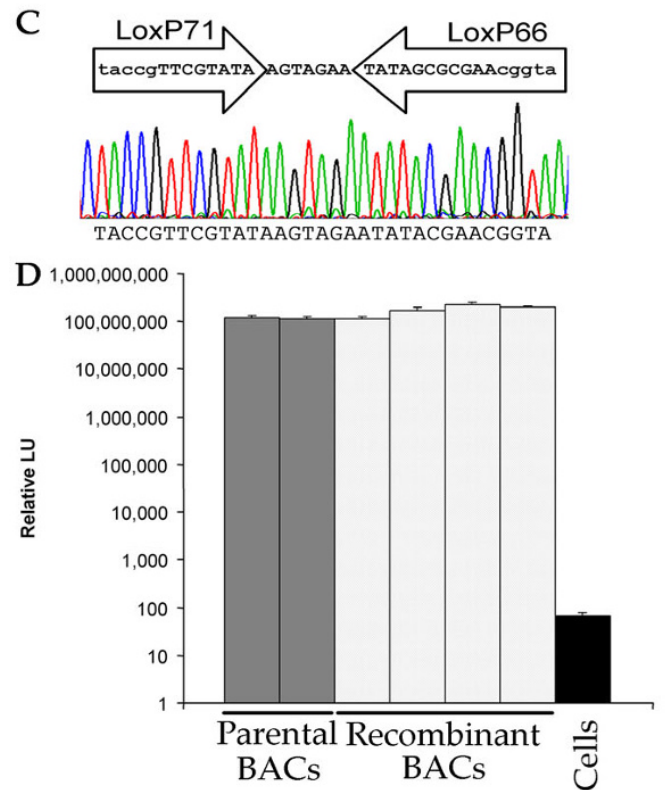

Figure 2

Selective removal of marker genes. A) Cartoon showing strategy for Recombineering of an expression cassette (expression) into the AcMNPV Bacmid DNA and selective removal of only the marker genes (selection) by Cre mediated recombination. B) PCR using primers labelled $a$ and $b$ in A using: Lanes I-2, two independent bacmid recombinants following red recombination; Lanes 3-6, four independent recombinants following Cre mediated recombination to remove the bacterial selectable markers; Lane 7, no template; Lane 8, unmodified bacmid DNA template; Lane 9, plasmid DNA template containing the selectable marker cassette; Lane I0, DNA marker. PCR products corresponding to the sizes predicted for the parental and recombinant products of the Cre mediated recombination are labelled $P$ and $R$ respectively. C) Sequencing trace file of a representative recombinant from the $\mathrm{PCR}$ analysis in $\mathrm{B}$ confirming the presence of a defective loxP incorporating the loxP7I and loxP66 arms that render the recombinant incapable of undergoing further Cre-mediated recombination. D) Renilla luciferase activity of the parental and recombinant bacmids from B when transfected into insect cells. Renilla luciferase activity was assayed at 48 hours post infection after on passage 2 of the recombinant virus. Background activity from uninfected cells is labelled Cells. 
A

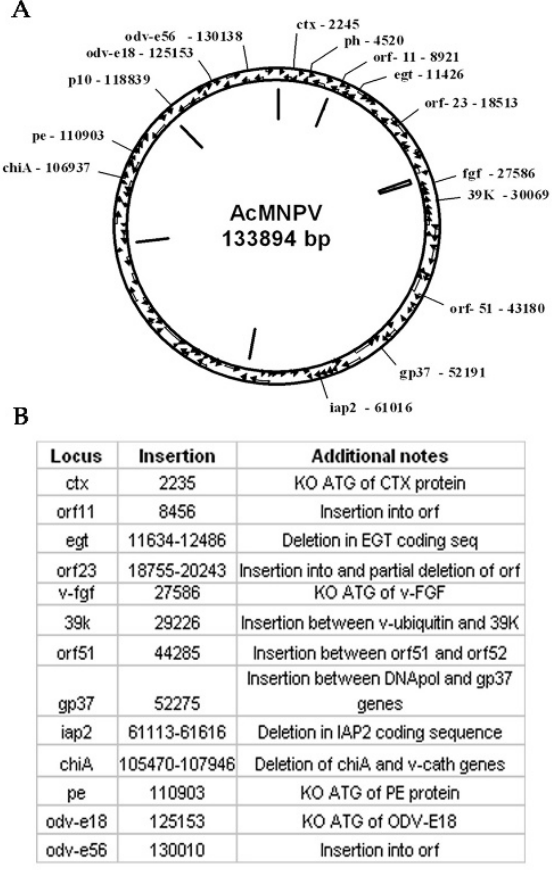

C
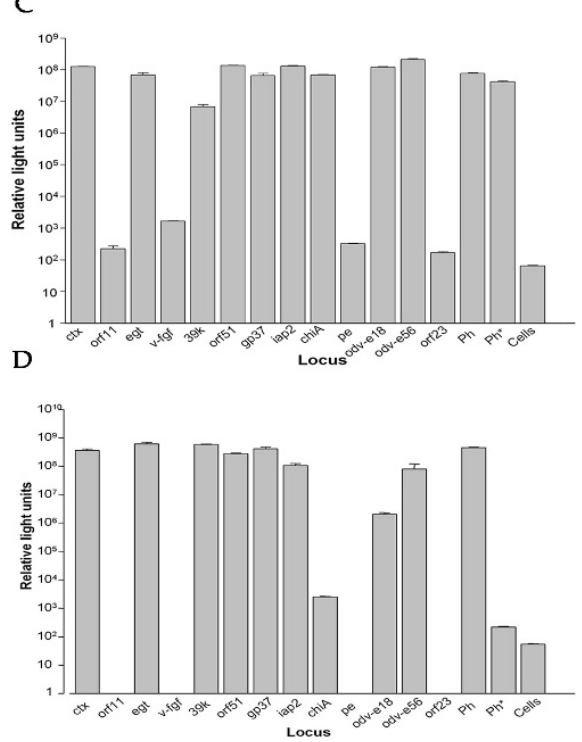

Figure 3

Identification of additional sites for expression of recombinant proteins in the AcMNPV genome. A) Genome of AcMNPV showing relative locations of loci used for protein expression. Bars inside the genome indicate position of $h r$ sequences. B) Table showing loci used for insertion and any additional changes made to locus. KO indicates knockout. C) Relative renilla luciferase activity at 48 hours post infection with virus modified to contain an additional firefly luciferase expression cassette at each locus indicated. All viruses had the same renilla luciferase expression cassette at the pl0 locus under control of the p35 promoter. Error bars indicted the standard deviation of five replicates for each locus. D) Normalised firefly luciferase activity showing relative expression of a polyhedrin promoter-polyhedrin terminator inserted at each locus as indicated. Error bars indicate the standard deviation from 5 replicates for each locus. Firefly luciferase was normalised using the renilla luciferase control expressed from the same genome. Firefly luciferase insertions at the orfI I, $v$-fgf, pe and orf 23 loci were excluded due to renilla luciferase levels more than 2 logs lower than positive control virus. Virus Ph has firefly luciferase at the polyhedrin locus and renilla luciferase at the plo locus. Virus $\mathrm{Ph}^{*}$ has the same plo renilla luciferase insertion but no firefly luciferase insertion. 
There was no clear correlation between the position of a locus within the virus genome and its ability to allow successful high level expression of the foreign reporter gene (Additional file 1: Figure S1). Nor was there any correlation between the level of expression observed for the reporter and that documented for the native gene at the same locus [30].

\section{Simultaneous co-expression of two and four genes to recover virus-like particles}

To confirm that the system could successfully express and correctly assemble protein complexes, two well defined virus-like particle (VLP) systems were used as models. VLPs were produced for influenza A virus by co-expressing the M1 and HA proteins of the SC35M strain of influenza [31]. M1 was first inserted at the egt locus and then, following removal of the bipartite cassette, the HA gene was inserted into the p10 locus (Fig. 4A). Expression of both proteins was confirmed by SDS-PAGE and Immunoblot analysis (Fig. 4A, left and middle panels) using antibody specific for Influenza A (H7N7). Furthermore, it was possible to purify influenza VLPs from the culture medium of Sf 9 cells infected with the recombinant baculovirus. The surface of these VLPs had the characteristic spike appearance of an enveloped virus (Fig. 4A, right panel) and it was possible to label these spike structures using immunogold labelling with anti-influenza (H7N7) antibody.

The other VLP made was for the non-enveloped orbivirus, bluetongue virus. VLPs for this virus require the coassembly of four proteins (VP2, VP3, VP5 and VP7). Expression of recombinant protein was confirmed by SDS-PAGE analysis (Fig. 4B, left panel) and VLPs with the characteristic morphology of mature BTV particles could be purified from insect cells infected with recombinant baculovirus (Fig. 4B, right panel).

\section{Coexpression of eight genes to recover the mouse CCT complex}

To extend our observations further, and to demonstrate the utility of the muli-locus expression system to the production of large mammalian protein complexes, the eight subunits of the mouse CCT chaperonin complex (CCT1 to CCT8, respectively) were each expressed from a different locus within the baculovirus genome (Fig. 5A.). All eight subunits were expressed and accumulated in insect cells to detectable levels, although there was a variation in the steady-state levels of protein between subunits. Expressed protein was detected with a polyclonal antibody against a conserved motif of the ATP-binding site of all CCT subunits (Fig. 5A middle panel). There was cross reaction between the antibody and some of the subunits of the endogenous insect CCT complex, and it was impossible to confirm the presence of CСТ8. To confirm mouse ССТ8 was expressed, a duplicate immunoblot was probed with
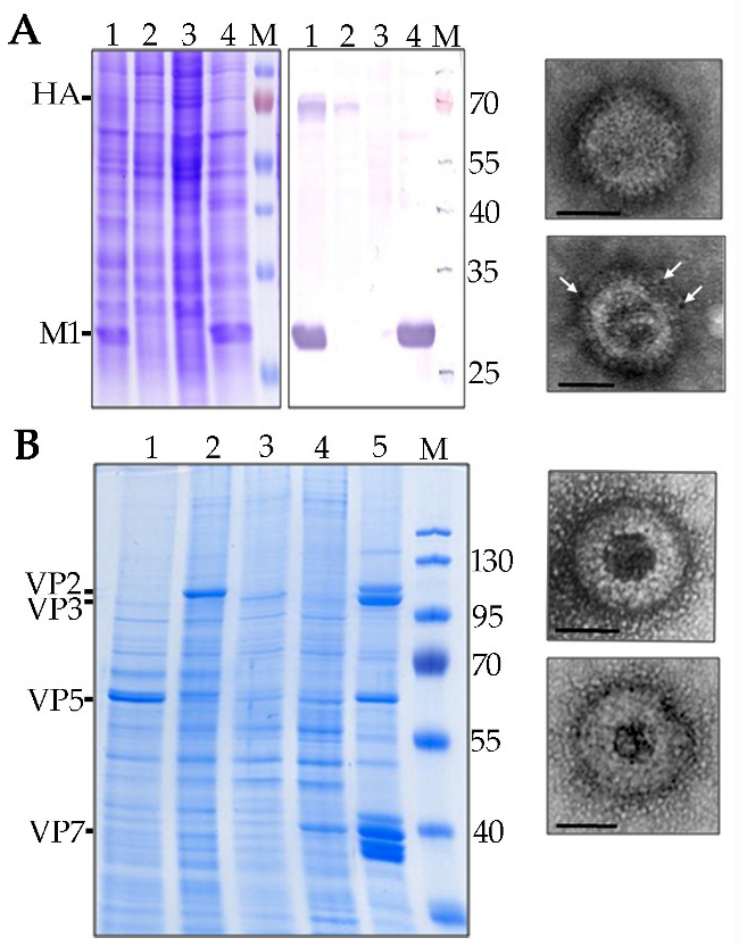

\section{Figure 4}

Expression of virus like particles. A) Influenza A VLPs. Left and middle, stained SDS-PAGE and immunoblot, respectively, of cells expressing; Lane I, Influenza HA (p/Olocus) and MI (egt locus); Lane 2, Influenza HA ( $p / 0$ locus) only; Lane 3, uninfected cells; Lane 4, Influenza MI (egt locus). The immunoblot was probed with a polyclonal anti-influenza (H7N7) antibody. Right panel negative stain electron micrographs of influenza VLPs purified from the culture medium of infected cells. Bar $=50 \mathrm{~nm}$. In the lower panel white arrows indicate the position of $5 \mathrm{~nm}$ gold particles labelling the HA spikes of the VLPs. B) Bluetongue virus VLPs. Left, stained SDS-PAGE of lysate from cells infected with AcMNPV expressing: Lane I, BTV-I VP5 (p/0 locus); Lane 2, BTVIVP2 (odv-e56 locus); Lane 3, BTV-I VP3 (polyhedrin locus); Lane 4, BTV-I VP7 (gp37 locus); Lane 5, Semipurified VLPs recovered from a virus coexpressing all 4 BTV proteins; $M$, marker lanes with proteins of defined mass. Right hand panels, electron micrographs of negatively stained BTV VLPs. Bar $=50 \mathrm{~nm}$.

a monospecific antibody to the CCT8 protein (Fig. 5A bottom panel). These data confirmed all eight CCT subunits were expressed using the multi-locus expression approach.

The native CCT chaperone complex consists of a hexadecamer arranged as two stacked rings of 8 subunits $[32,33]$. To confirm formation of a complex for the recombinant mouse CCT subunits, we performed ratezonal centrifugation in $10-40 \%(\mathrm{w} / \mathrm{w})$ sucrose gradients. The characteristic protein profile for the CCT complex was 


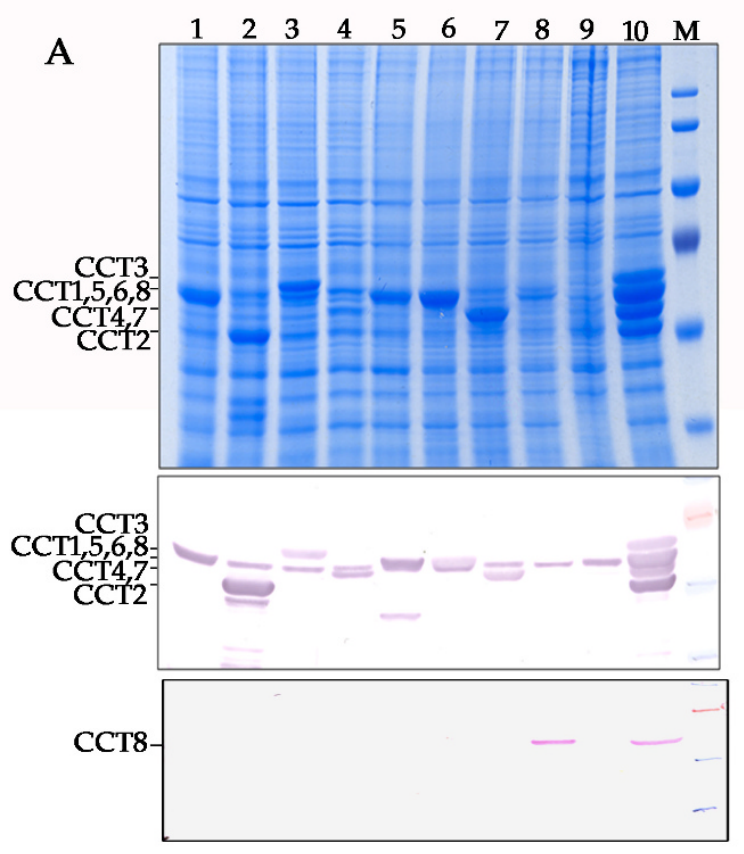

B

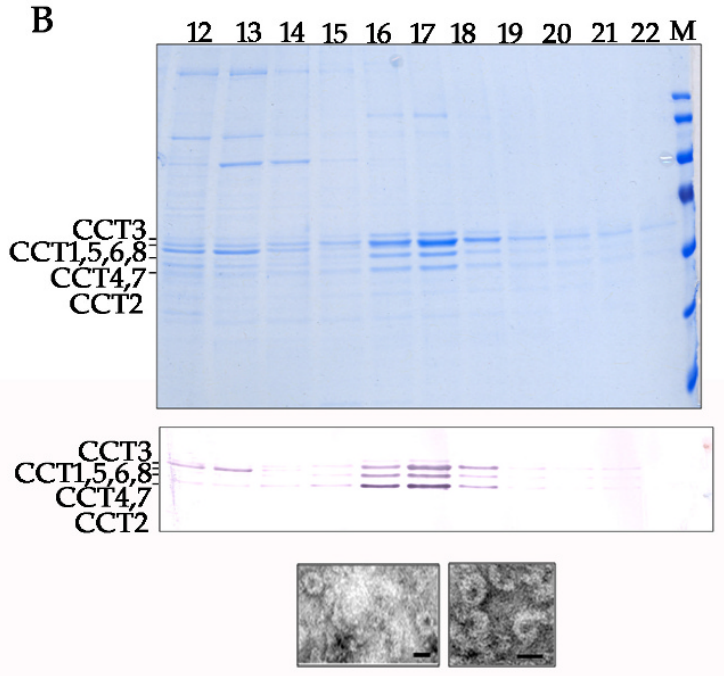

Figure 5

Recovery of recombinant mouse CCT complex by co-expression of 8 proteins in the same insect cell. A) Top panel, cell lysates from cells expressing mouse CCT subunits I-8 (lanes I-8, respectively). Lane 9, uninfected cells; Lane I0, lysate from cells coexpressing the CCT subunits from the same baculovirus genome; M, marker protein lane. Relative migration of CCT subunits is indicated on the left hand side of the gel. Loci used in the expression were CCTI-polyhedrin, CCT2p 10, CCT3-egt, CCT4-39K, CCT5-gp37, CCT6-odv-e56, CCT7-ctx, CCT8-orf5I. Middle panel, Immunoblot using pan-specific antiserum against mouse CCT, sample order as in top panel. Bottom panel, Immunoblot using monospecific antiserum against mouse CCT theta (CCT8), sample order as in top panel. B) Fractions 12 to 22 (of 34) numbered from the top of a $10 \%$ $40 \%(w / w)$ sucrose gradient. CCT complex proteins peak in the expected ratio in fractions 16 and 17. Relative migrations expected for the CCT proteins are indicated on the left of the gel. Middle panel, immunoblot using pan-specific antiserum against mouse CCT subunits. Lower panel, electron micrographs of negatively stained sample from the $23.5 \mathrm{~S}$ peak fraction collected from the sucrose gradient. Ring structures consistent with CCT complexes can be detected. Bar $=25 \mathrm{~nm}$. 
detected as a peak following centrifugation (Fig. 5B, top panel lanes 16-17). The identity of these proteins as CCT subunits was confirmed by immunoblotting (Fig. 5B, middle panel). The averaged migration of this complex in the gradient over 3 experiments was consistent with a sedimentation coefficient of $23.5+/-1.1 \mathrm{~S}$ and was similar to that reported for the native mouse CCT complex[32]. Finally, fractions corresponding with the $23.5 \mathrm{~S}$ recombinant mouse CCT complex were visualised by negative staining and electron microscopy (Fig. 5B, bottom panel). It was possible to visualise ring-like structures consistent with those reported with the mouse СCT[32].

\section{Discussion}

This report demonstrates that lambda red recombination can be used to efficiently engineer high level expression of recombinant protein and protein complexes from the AcMNPV genome. Using a new bipartite selection system up to 88 fold improvement $(\mathrm{p}=0.03)$ in recovery of recombinants was achieved. All putative recombinants recovered using the bipartite selection were subsequently confirmed to be correctly modified. This is a substantial improvement over the single antibiotic selection $[21,28]$ which in our hands produced a high level of false positive recombinants (recombinants that were apparently chloramphenicol resistant but subsequently did not have the target insertion). Furthermore, there was no requirement to use PCR generated linear DNA for recombination, which avoids potential problems associated with mutations introduced by repeated PCR of coding regions. To our knowledge the bipartite selection in this report has not previously been applied to recombination in baculovirus, although there have been reports of the zeocin resistance gene alone being used for knock out of baculovirus genes by lambda red recombination[34].

By flanking the $Z e o^{R}$-LacZ $\alpha$ selection with self inactivating loxp sites it was possible to re-use the selectable markers and carry out iterative selection at multiple loci within the baculovirus genome (Fig. 2). This iterative process itself allows the potential for combining several different subunits into the same baculovirus genome in different combinations without having to generate a new multi-gene transfer vector for every combination of proteins that is to be expressed.

Despite the extensive protein expression work that has been undertaken in the baculovirus system most expression has focussed on replacement of the polyhedrin or p10 genes. Relatively little literature describes the use of alternative loci for the expression of recombinant protein [10,35-37]. This study identifies seven genetic loci (ctx, egt, 39k, orf51, gp37, iap2 and odv-e56), in addition to polyhedrin and $p 10$, suitable for high level expression of foreign genes.
For the loci that did not give good expression, four (orf11, $v-f g f$, pe and orf23) also resulted in a reduced expression of a separate marker protein present in all recombinants. This suggests that these insertions resulted in impairment of functions essential for virus replication or gene expression. The low level expression of firefly luciferase expression in the chiA and odv-e18 insertion viruses was unexpected for different reasons. Other reports have recorded insertion of recombinant protein expression cassettes into the chiA locus $[10,11]$. It is possible that the reduced level of firefly luciferase expression from this locus in our experiments was due to effects on genes flanking the insertion. For $o d v$-e18, recent reports based on deletion within the coding sequence of $o d v-e 18$ and the upstream flanking gene have suggested that this protein is essential for budded virus production and cell to cell movement $[38,39]$. In our experiments, where odv-e18 was inactivated by specific mutation of the start codon and by insertion of the firefly luciferase cassette, there was no evidence from renilla luciferase expression of impairment of the ability of this mutant virus to replicate or spread cell to cell. However, given the $\sim 2 \log$ reduction in firefly luciferase levels compared to virus without this insertion, we cannot rule out the possibility that a small population of virus in which the mutation had reverted was complementing the virus expressing the reporter gene.

To confirm the utility of the system for the expression of recombinant protein complexes two-protein and fourprotein virus like particles (VLPs) were produced to influenza A and bluetongue virus, respectively. VLPs for both these viruses are effective immunogens against the respective pathogen [40-42] and thus represent potential biotechnological applications of the multi-locus expression approach. Both VLPs were formed normally and could be purified from the culture medium (for influenza VLPs), or cells (for BTV), of baculovirus infected cultures. To extend these observations further and to demonstrate the usefulness of the approach to low abundance mammalian protein complexes we expressed all eight subunits of the mouse CCT complex. This complex is usually purified from testes or by large scale fermentation culture of yeast $[32,43,44]$. However a reliable source of large quantities of correctly folded recombinant mammalian CCT complex was not previously available. It was possible to recover between 1 and $6 \mathrm{mg} / \mathrm{L}$ culture of 23.5S CCT complex. To our knowledge this is the first report of the successful expression of mammalian CCT chaperone from a heterologous system, and offers for the first time the possibility of mutational analysis of this essential machinery involved in cytoskeletal protein folding and cell cycle control.

In addition to providing the opportunity for assembly of multiprotein complexes, the multilocus system also offers 
the opportunity to test and manipulate relative expression of each subunit of a complex independently, without any need for recloning into different transfer vectors, and for swapping subunits between complexes, also without need for recloning. Thus, in terms of flexibility, it offers substantial improvement over the current approaches for baculovirus multi-protein complex expression.

\section{Conclusion}

1. Use of bipartite selections can significantly improve selection of modified bacterial artificial chromosomes carrying baculovirus DNA. Furthermore this approach is sufficiently robust to allow routine modification of the virus genome. 2 . In addition to the commonly used $p 10$ and polyhedrin loci, the ctx, egt, 39k, orf51, gp37, iap2 and odv-e56 loci in AcMNPV are all suitable for the high level expression of heterologous genes. 3. Two protein, four protein and eight protein complexes including virus-like particles and cellular chaperone complexes can be produced using the new approach.

\section{Methods}

\section{Cell Lines and Virus}

Sf9 and $S f 21$ cells were cultured at $28^{\circ} \mathrm{C}$ in Insect-Xpress (Lonza, Basel, Switzerland) and TC100 media (Biochrom AG, Berlin, Germany), respectively. All cells were routinely cultured in the presence of penicillin $(100 \mathrm{U}) / \mathrm{strep}-$ tomycin $(100 \mu \mathrm{g} / \mathrm{ml}) /$ Amphotericin $\mathrm{B}(250 \mathrm{ng} / \mathrm{ml})$ (Sigma-Aldrich Chemical Co., St. Louis, Mo.). TC100 medium was supplemented with $10 \%$ Foetal bovine serum. Viruses used in this study were based on the cloned AcMNPV genome [3]. For recombinants where protein was expressed from the polyhedrin locus, the BAC10:KO1629 bacmid [28] was used, for all other expressions a modified version of the bacmid bMON14272 [3] in which the expression of the $L a c Z \alpha$ fragment had been inactivated was used.

\section{Antibodies}

Polyclonal anti influenza H7N7 antibody was a gift from H.D. Klenk (University of Marburg). The pan-CCT antibody, UM1, and the monospecific CCT8 antibody used were as described [45]. Anti rabbit immunogold antibody was purchased from Sigma-Aldrich. Negative staining was with $2 \%(\mathrm{w} / \mathrm{v})$ uranyl acetate. All immunoblotting was performed using Immobilon $\mathrm{P}$ membrane (Millipore, Billerica, MA) according to the manufacturer's instructions.

\section{Construction of transfer vectors}

Base expression and selection cassettes with the structure detailed in Fig. 1A, but without flanking AcMNPV were constructed in pBluescript SK (Stratagene, Cedar Creek, Texas). Transfer vectors targeting the different genetic loci within baculovirus were constructed by the general strategy of amplification and cloning of the corresponding region of the AcMNPV genome followed either by mutagenesis to introduce a restriction enzyme site, allowing insertion of the base expression and selection cassette, or digestion with restriction enzymes to introduce the same cassette. Details of the primers and restriction enzymes used for each locus are detailed in table 1. All constructs were designed such that a linear DNA fragment containing the AcMNPV flanking sequences and the expression and selection cassettes could be released by digestion with BsaI. Where possible, for each locus the expression and selection cassettes were inserted in both orientations and firefly luciferase monitored independently in each orien-

Table I: Primers used for amplification of sequences for each AcMNPV locus used and restriction enzyme site used for insertion of reporter and expression cassettes.

\begin{tabular}{|c|c|c|c|}
\hline Locus & Forward Primer & Reverse primer & Restriction enzymes used \\
\hline$c t x$ & CACTTGACTCGATTGCGCG & TATTTATTGTCTACATGAACACG & $\mathrm{m}^{*} E c o R V$ \\
\hline orfl I & GTTGCACCTTTGACGAAGCGG & TCACAATCCATAACACACAACAGG & $\mathrm{m}^{*}$ EcoRV \\
\hline egt & ATGTGCGACCATTGTTGGGC & GTTGTCACATCTGACTACTCC & pd \\
\hline orf23 & AGAGTGCGTTAATCTGTACACC & ATCATAGGGTACAACACAGG & Stul/Sfol \\
\hline$v$-fgf & CCGGCAAAATCAAAGCGAGC & GATTACACGTGACATTTACGATGG & $\mathrm{m}^{*}$ EcoRV \\
\hline $39 k$ & CCTGGTAATTTTTGACCACG & CGCAGCAATTCCAGCGAGC & $\mathrm{m}^{*} E \mathrm{coRV}$ \\
\hline orf5 I & AAATGACTAGACAAGAAATTGCC & AGTTGTACAAATCACAAATATAAAAG & $\mathrm{BmgBI}$ \\
\hline$g p 37$ & ATTGACGGGCCGTCGGCACG & CGATCATGCAAAAGTACATGC & $\mathrm{m}^{*}$ EcoRV \\
\hline iap2 & CCGCGGCTAAGCGTTAAACC & TTCGAATACGTGTGTCGTTTAATTTGC & BstBI/Sacll \\
\hline chiA & TAAACGCTCCGACTCTGTGG & CGAGGGCCGCGGCCAGTGGGTC & pd \\
\hline pe & GCATTTTTCCAATGTGGTAGACG & CTTTAGCGGTTTCCAACGCC & $\mathrm{m}^{*}$ EcoRV \\
\hline odv-el 8 & TCTCAAACACGGTGCCTGC & TCGTTGGTTTCAGTGACCAC & $\mathrm{m}^{*}$ EcoRV \\
\hline odv-e56 & CAACATGACGCCGCTGCCG & TTATCGAGGGGCCGTTGTTG & $\mathrm{m}^{*} E \mathrm{coRV}$ \\
\hline
\end{tabular}

$\mathrm{m} * \mathrm{EcoRV}$, indicates that an $E c o R V$ site was engineered by site directed mutagenesis using the quickchange site directed muagenesis system (Stratagene) according to the manufacturer's directions. Pd indicates that a deletion was engineered by PCR. In the case of the egt locus this deletion removes the equivalent of $n t$ II622-12474 nt in the AcMNPV genome. For chiA the engineered deletion removes the chiA and cathepsin genes and is positioned nt 10546I-107946 nt in the AcMNPV genome. 
tation. For protein expression experiments the coding sequence of each gene was amplified from a cDNA clone of the respective gene and cloned downstream of the polyhedrin promoter into the same vectors. For CCT the complete set of mouse CCT cDNAs [46] were used.

\section{Luciferase assays}

All luciferase assays were completed using the Dual Luciferase Reporter Assay system (Promega, Madison, USA) using a Turner Biosciences 20/20 n luminometer according to the manufacturer's instructions.

\section{Lambda red recombination in $\mathrm{E}$. coli}

All bacmids were maintained in the E. coli strain EL350 [47] which was grown at $32{ }^{\circ} \mathrm{C}$. Expression of the $\lambda$ bet, exo and gam genes in this lysogen were induced by incubation at $42^{\circ} \mathrm{C}$ for a 30 minute period immediately harvesting cells for the preparation of batches of cells for electroporation. Once prepared, electrocompetent cells were stored at $-80^{\circ} \mathrm{C}$ prior to use. For lambda red recombination 30 ng linear DNA was introduced into recombination competent cells by electroporation. Cells were grown for two hours at $32^{\circ} \mathrm{C}$ and then plated under Kanamycin $(50 \mu \mathrm{g} /$ $\mathrm{ml})$ Zeocin $(15 \mu \mathrm{g} / \mathrm{ml})$ and IPTG/X-gal $(0.2 \mathrm{mM}$ and 40 $\mu \mathrm{g} / \mathrm{ml}$ respectively). For modification of BAC10:KO1629 chloramphenicol selection $(15 \mu \mathrm{g} / \mathrm{ml})$ was also included. Plates were incubated at $32^{\circ} \mathrm{C}$ and blue colonies picked to seed liquid cultures after 24 hours. All preparation of DNA was performed according to standard molecular techniques [48].

\section{Cre recombination in E. coli}

Expression of Cre recombinase in EL350 was induced by treatment of mid-log phase cultures with $0.1 \%$ Arabinose for 2 hours followed by a change of media and outgrowth for 16 hours at $32^{\circ} \mathrm{C}$. Cells were then plated on selective media containing IPTG $(0.2 \mathrm{mM}), \mathrm{X}$-Gal $(40 \mu \mathrm{g} / \mathrm{m})$ and kanamycin $(50 \mu \mathrm{g} / \mathrm{ml})$. White colonies were re-streaked onto the same selection after 24 hours and then used to seed liquid cultures.

\section{Purification of VLPs}

Bluetongue VLPs were purified from infected $S f 9$ cells as described [7]. Influenza A (HA/M1) VLPs were purified from the culture medium of infected $S f 9$ cells at 72 hours post infection. Culture medium was clarified by spinning at $9500 \times \mathrm{g}$ for 10 minutes at $4^{\circ} \mathrm{C}$ before being spun onto a $60 \% \mathrm{w} / \mathrm{v}$ sucrose cushion for 1 hour at $90,000 \times g$ at $4{ }^{\circ} \mathrm{C}$ in a SW28 rotor to concentrate VLPs. Material on top of the sucrose cushion was then diluted six fold in PBS and loaded onto a $20 \%-30 \%-60 \%(\mathrm{w} / \mathrm{w})$ sucrose step gradient before being spun again for 1 hour at $90,000 \times g$ at $4{ }^{\circ} \mathrm{C}$ in a SW28 rotor. Material at the $30-60 \%$ sucrose interface contained the particles shown in Fig. 5.

\section{Purification of CCT}

Sf21 cells were infected with recombinant baculovirus at multiplicity of infection of 3 and harvested at 48-72 hours post infection. Cells were washed in PBS and lysed at room temperature in lysis buffer (50 mM HEPES pH8.0, $100 \mathrm{mM} \mathrm{NaCl}, 0.05 \%$ (v/v) Tergitol NP-9). Nuclei were pelleted at $1000 \times g$ for 5 minutes then cell debris pelleted at $9500 \times \mathrm{g}$ for 10 minutes at $4^{\circ} \mathrm{C}$. Clarified cell lysate was spun at $100,000 \times g$ at $4^{\circ} \mathrm{C}$ for 1 hour then serially filtered through $0.5 \mu \mathrm{m}, 0.2 \mu \mathrm{m}$ and $0.05 \mu \mathrm{m}$ filters (Pall, East Hills, NY and Spectrum Laboratories, Breda, The Netherlands). Filtered lysate was concentrated using a centriplus filtration device (Millipore, Billerica, MA) with a $100 \mathrm{kDa}$ cut-off. Concentrated lysate was loaded onto 10-40\% (w/ $\mathrm{w})$ continuous sucrose gradients in $50 \mathrm{mM}$ Hepes pH8.0, $100 \mathrm{mM} \mathrm{NaCl}$ and spun for 16 hours at $4^{\circ} \mathrm{C}$ at 30,000 $\mathrm{rpm}$ in a SW40 rotor. Following centrifugation $500 \mu \mathrm{l}$ fractions were collected from the bottom of the tube.

\section{Authors' contributions}

RN conceived the project, designed the experiments, carried out the research, and wrote the paper. MS, MB and CC made transfer plasmids for the expression of BTV VP3, VP5 and VP7, respectively. KW provided cDNA and antibodies for mouse CCT. He also provided substantial guidance on the best purification strategies for this protein complex. PR contributed to the design of experiments, provided expertise for purification of bluetongue VLPs and secured funding for the project. All authors were involved in the revision of the draft manuscript and have agreed the final content.

\section{Additional material}

\section{Additional file 1}

Figure S1. PDF file containing supplementary figure.

Click here for file

[http://www.biomedcentral.com/content/supplementary/14712199-10-87-S1.pdf]

\section{Acknowledgements}

This work was supported by the Biotechnology and Biological Sciences Research Council, UK. The authors would like to gratefully acknowledge the gift of cDNA for influenza A SC35M and polyclonal antibody from H.D. Klenk (University of Marburg), the E. coli strain EL350 from C.T. Dolphin (King's College, London), and the bacmid BACI0:KOI629 from I.M. Jones (University of Reading).

\section{References}

I. Summers MD: Milestones leading to the genetic engineering of baculoviruses as expression vector systems and viral pesticides. Adv Virus Res 2006, 68:3-73.

2. Ayres MD, Howard SC, Kuzio J, Lopez-Ferber M, Possee RD: The complete DNA sequence of Autographa californica nuclear polyhedrosis virus. Virology 1994, 202(2):586-605. 
3. Luckow VA, Lee SC, Barry GF, Olins PO: Efficient generation of infectious recombinant baculoviruses by site-specific transposon-mediated insertion of foreign genes into a baculovirus genome propagated in Escherichia coli. J Virol 1993, 67(8):4566-4579.

4. Smith GE, Summers MD, Fraser MJ: Production of human beta interferon in insect cells infected with a baculovirus expression vector. Mol Cell Biol 1983, 3(12):2156-2165.

5. Kitts PA, Possee RD: A method for producing recombinant baculovirus expression vectors at high frequency. Biotechniques 1993, 14(5):810-817.

6. Emery VC, Bishop DH: The development of multiple expression vectors for high level synthesis of eukaryotic proteins: expression of LCMV-N and AcNPV polyhedrin protein by a recombinant baculovirus. Protein Eng 1987, I(4):359-366.

7. French TJ, Marshall JJ, Roy P: Assembly of double-shelled, viruslike particles of bluetongue virus by the simultaneous expression of four structural proteins. I Virol 1990 64(I 2):5695-5700.

8. Latham T, Galarza JM: Formation of wild-type and chimeric influenza virus-like particles following simultaneous expression of only four structural proteins. J Virol 200I, 75(I3):6154-6|65.

9. Pushko P, Bray M, Ludwig GV, Parker M, Schmaljohn A, Sanchez A, Jahrling PB, Smith JF: Recombinant RNA replicons derived from attenuated Venezuelan equine encephalitis virus protect guinea pigs and mice from Ebola hemorrhagic fever virus. Vaccine 2000, I9(I): 142-153.

10. Berger I, Fitzgerald DJ, Richmond TJ: Baculovirus expression system for heterologous multiprotein complexes. Nat Biotechnol 2004, 22(I 2): | 583-1587.

II. Fitzgerald DJ, Berger P, Schaffitzel C, Yamada K, Richmond TJ, Berger I: Protein complex expression by using multigene baculoviral vectors. Nat Methods 2006, 3(1 2): 1021-1032

12. French TJ, Roy P: Synthesis of bluetongue virus (BTV) corelike particles by a recombinant baculovirus expressing the two major structural core proteins of BTV. I Virol 1990, 64(4): $1530-1536$.

13. Weyer U, Possee RD: A baculovirus dual expression vector derived from the Autographa californica nuclear polyhedrosis virus polyhedrin and $\mathrm{p} I 0$ promoters: co-expression of two influenza virus genes in insect cells. J Gen Virol 1991, 72(Pt I 2):2967-2974.

14. Bertolotti-Ciarlet A, Ciarlet M, Crawford SE, Conner ME, Estes MK: Immunogenicity and protective efficacy of rotavirus 2/6virus-like particles produced by a dual baculovirus expression vector and administered intramuscularly, intranasally, or orally to mice. Vaccine 2003, 2I (25-26):3885-3900.

15. Kamita SG, Maeda S, Hammock BD: High-frequency homologous recombination between baculoviruses involves DNA replication. J Virol 2003, 77(24): |3053-I306I.

16. Crouch EA, Passarelli AL: Genetic requirements for homologous recombination in Autographa californica nucleopolyhedrovirus. J Virol 2002, 76( 18):9323-9334.

17. Mikhailov VS, Okano K, Rohrmann GF: Baculovirus alkaline nuclease possesses a $5^{\prime}-$-.> $3^{\prime}$ exonuclease activity and associates with the DNA-binding protein LEF-3. J Virol 2003, 77(4):2436-2444.

18. Pijlman GP, Born E van den, Martens DE, Vlak JM: Autographa californica baculoviruses with large genomic deletions are rapidly generated in infected insect cells. Virology 200I 283(I):132-138.

19. Pijlman GP, van Schijndel JE, Vlak JM: Spontaneous excision of BAC vector sequences from bacmid-derived baculovirus expression vectors upon passage in insect cells. J Gen Virol 2003, 84(Pt I0):2669-2678

20. Datsenko KA, Wanner BL: One-step inactivation of chromosomal genes in Escherichia coli K-12 using PCR products. Proc Natl Acad Sci USA 2000, 97 ( 12 2):6640-6645.

21. Lin G, Blissard GW: Analysis of an Autographa californica nucleopolyhedrovirus lef-I I knockout: LEF-I I is essential for viral DNA replication. J Virol 2002, 76(6):2770-2779.

22. Vanarsdall AL, Pearson MN, Rohrmann GF: Characterization of baculovirus constructs lacking either the Ac I0I, Ac I42, or the Ac 144 open reading frame. Virology 2007, 367(I): | $87-195$.
23. Fang M, Dai X, Theilmann DA: Autographa californica multiple nucleopolyhedrovirus EXONO (ORFI4I) is required for efficient egress of nucleocapsids from the nucleus. J Virol 2007, 8 I ( I 8):9859-9869.

24. Yamagishi J, Burnett ED, Harwood SH, Blissard GW: The AcMNPV pp3 I gene is not essential for productive AcMNPV replication or late gene transcription but appears to increase levels of most viral transcripts. Virology 2007, 365(I):34-47.

25. Stewart TM, Huijskens I, Willis LG, Theilmann DA: The Autographa californica multiple nucleopolyhedrovirus ie0iel gene complex is essential for wild-type virus replication, but either IE0 or IEI can support virus growth. J Virol 2005, 79(8):4619-4629.

26. Kamita SG, Nagasaka K, Chua JW, Shimada T, Mita K, Kobayashi M, Maeda S, Hammock BD: A baculovirus-encoded protein tyrosine phosphatase gene induces enhanced locomotory activity in a lepidopteran host. Proc Natl Acad Sci USA 2005, I 02(7):2584-2589.

27. Milks ML, Washburn JO, Willis LG, Volkman LE, Theilmann DA: Deletion of pe38 attenuates AcMNPV genome replication, budded virus production, and virulence in heliothis virescens. Virology 2003, 310(2):224-234.

28. Zhao Y, Chapman DA, Jones IM: Improving baculovirus recombination. Nucleic Acids Res 2003, 3 I (2):E6-6.

29. Albert H, Dale EC, Lee E, Ow DW: Site-specific integration of DNA into wild-type and mutant lox sites placed in the plant genome. Plant J 1995, 7(4):649-659.

30. Jiang SS, Chang IS, Huang LW, Chen PC, Wen CC, Liu SC, Chien LC, Lin CY, Hsiung CA, Juang JL: Temporal transcription program of recombinant Autographa californica multiple nucleopolyhedrosis virus. J Virol 2006, 80( I 8):8989-8999.

31. Scheiblauer H, Kendal AP, Rott R: Pathogenicity of influenza A/ Seal/Mass/I/80 virus mutants for mammalian species. Arch Virol 1995, I40(2):34I-348

32. Lewis VA, Hynes GM, Zheng D, Saibil H, Willison K: T-complex polypeptide- $I$ is a subunit of a heteromeric particle in the eukaryotic cytosol. Nature 1992, 358(6383):249-252.

33. Liou $A K$, Willison KR: Elucidation of the subunit orientation in CCT (chaperonin containing TCPI) from the subunit composition of CCT micro-complexes. EMBO J 1997, 16(14):431I-4316.

34. Dai X Stewart TM, Pathakamuri JA, Li O, Theilmann DA Autographa californica multiple nucleopolyhedrovirus exon0 (orfl4I), which encodes a RING finger protein, is required for efficient production of budded virus. J Virol 2004, 78(18):9633-9644

35. Belyaev AS, Hails RS, Roy P: High-level expression of five foreign genes by a single recombinant baculovirus. Gene 1995, I 56(2):229-233.

36. Vlak JM, Schouten A, Usmany M, Belsham GJ, Klinge-Roode EC, Maule A], Van Lent JW, Zuidema D: Expression of cauliflower mosaic virus gene I using a baculovirus vector based upon the pI0 gene and a novel selection method. Virology 1990, I79(I):3।2-320.

37. Braunagel SC, Elton DM, Ma H, Summers MD: Identification and analysis of an Autographa californica nuclear polyhedrosis virus structural protein of the occlusion-derived virus envelope: ODV-E56. Virology 1996, 217(I):97-II0

38. McCarthy CB, Dai X, Donly C, Theilmann DA: Autographa californica multiple nucleopolyhedrovirus acl 42 , a core gene that is essential for BV production and ODV envelopment. Virology 2008, 372(2):325-339.

39. McCarthy CB, Theilmann DA: AcMNPV acl43 (odv-el8) is essential for mediating budded virus production and is the 30th baculovirus core gene. Virology 2008, 375(I):277-29I.

40. Roy P, Bishop DH, LeBlois $\mathrm{H}$, Erasmus BJ: Long-lasting protection of sheep against bluetongue challenge after vaccination with virus-like particles: evidence for homologous and partial heterologous protection. Vaccine 1994, I 2(9):805-8II.

4I. Roy P, French T, Erasmus B]: Protective efficacy of virus-like particles for bluetongue disease. Vaccine 1992, I0(I):28-32.

42. Galarza JM, Latham T, Cupo A: Virus-like particle vaccine conferred complete protection against a lethal influenza virus challenge. Viral Immunol 2005, I 8(2):365-372.

43. Frydman J, Nimmesgern E, Erdjument-Bromage $H$, Wall JS, Tempst $P$, Hartl FU: Function in protein folding of TRiC, a cytosolic ring 
complex containing TCP-I and structurally related subunits. $E M B O$ J 1992, I I(I3):4767-4778.

44. Pappenberger G, McCormack EA, Willison KR: Quantitative actin folding reactions using yeast CCT purified via an internal tag in the CCT3/gamma subunit. J Mol Biol 2006, 360(2):484-496.

45. Hynes G, Kubota H, Willison KR: Antibody characterisation of two distinct conformations of the chaperonin-containing TCP-I from mouse testis. FEBS Lett 1995, 358(2): I29-132.

46. Kubota H, Hynes G, Carne A, Ashworth A, Willison K: Identification of six Tcp-I-related genes encoding divergent subunits of the TCP-I-containing chaperonin. Curr Biol I994, 4(2):89-99.

47. Lee EC, Yu D, Martinez de Velasco J, Tessarollo L, Swing DA, Court DL, Jenkins NA, Copeland NG: A highly efficient Escherichia coli-based chromosome engineering system adapted for recombinogenic targeting and subcloning of BAC DNA. Genomics 200I, 73(I):56-65.

48. Sambrook J, Russell DW: Molecular Cloning: A Laboratory Manual. Third edition. Cold Spring Harbor, New York: Cold Spring Harbor Laboratory Press; 200I.

Publish with Bio Med Central and every scientist can read your work free of charge

"BioMed Central will be the most significant development for disseminating the results of biomedical research in our lifetime. "

Sir Paul Nurse, Cancer Research UK

Your research papers will be:

- available free of charge to the entire biomedical community

- peer reviewed and published immediately upon acceptance

- cited in PubMed and archived on PubMed Central

- yours - you keep the copyright 